\title{
Gold Stability and Diffusion in the Au/TS-1 Catalyst
}

Brandon C. Bukowski*, W. Nicholas Delgass, Jeffrey Greeley

Charles D. Davidson School of Chemical Engineering, Purdue University, West Lafayette IN 47907

\section{Supporting Information}

\section{S1 Supplementary Figures}
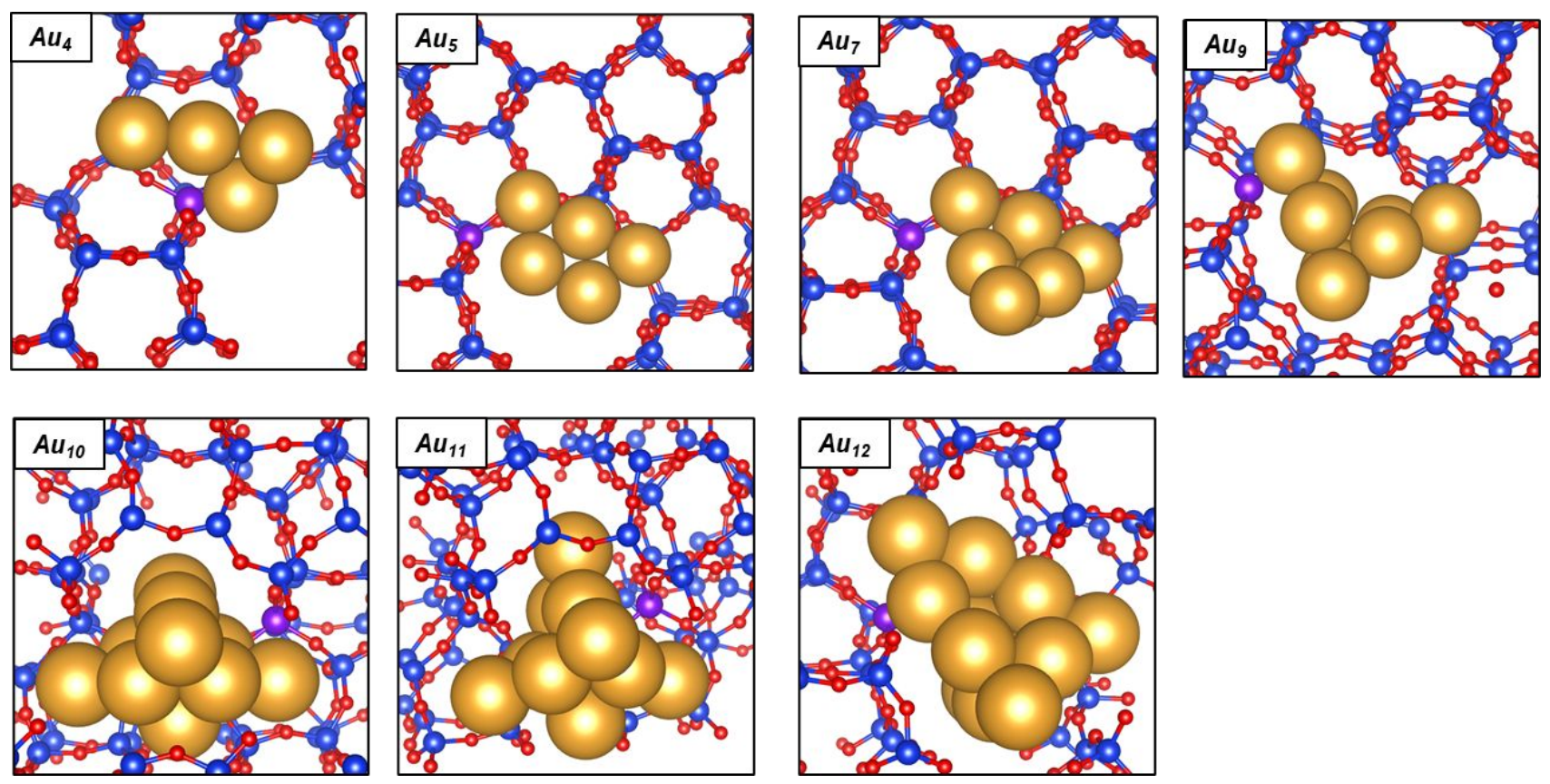

Figure S1 Representative lowest energy gold clusters for those not included in Figure 2.

\section{S2 Supplementary Discussion}

\section{S21 Dynamic Correction effects on predicted diffusion coefficients}

Transition state theory may overestimate diffusion coefficients when correlated crossings of the reaction coordinate are not considered due to rapid re-crossings during diffusion. ${ }^{1-3}$ The dynamic correction factor accounts for the fraction of barrier crossing attempts that successfully reach the product basin within a correlation timespan. For a dynamic correction factor (DCF) of $\sim 1$, all barrier-crossing attempts reach the product state and the dynamically corrected rate constant is equal to the standard rate constant discussed in the main text. This behavior is observed at low loadings for alkanes in the LTA zeolite, but can deviate significantly at higher adsorbate loadings. ${ }^{1}$ DCF values can deviate depending on other factors as well, such as certain pore geometries. To evaluate the DCF, the transition state surface must be determined (such as accomplished here), 
and additional simulations are performed where the adsorbates are initialized on the transition state surface and given a random initial velocity from the corresponding Boltzmann distribution. This technique is exceptionally difficult using ab-initio $\mathrm{MD}$ in a periodic lattice due to the computer time required. While it is not possible to quantitatively determine DCFs for the Au/TS-1 catalyst, we can make a few reasonable observations. First, the loading of gold was fixed to one cluster (of varying size) per simulation box. Second, the free energy surface local to the transition state surface was sharp for the trimer and tetramer gold clusters. Third, gold clusters have significant mass and diffuse at significantly slower timescales than most alkanes or single atoms. Based on these observations and the inability to quantify DCFs directly, we make the reasonable assumption that DCFs are $\sim 1$ for all gold clusters and re-crossing events are minimal.

\section{S22 Comparisons with Knudsen diffusion}

For smaller pore solids, the mean free path of an adsorbate may be longer than the pore size and frequent diffuse collisions result in a Fickian stochastic diffusion process. ${ }^{4}$ This allows for an expression for the diffusion coefficient dependent only on the temperature, adsorbate mass, and pore size. In MFI, the pores are $\sim 5.5 \AA$ and similar in size to the larger gold clusters. For micropores, it is more reasonable to expect molecular diffusion governed by the local free energy gradient and consequently transition state theory rather than Knudsen diffusion processes that are expected for mesopores $\sim 20 \AA$. We provide the comparison with Knudsen diffusion as an simple model that captures the inverse square root dependence of the molecular weight to outline that even for the large mass gold clusters, the particle mass is not so significant that it prevents diffusion entirely.

Another comparison for diffusion in porous materials is to consider the Knudsen diffusion coefficient. In Knudsen diffusion, gas molecules in a narrow pore diffuse through frequent collisions with pore walls, which is smaller than the mean free path of the gas. Knudsen diffusion is typically considered valid for the mesopore region with pore diameters greater than $\sim 20 \AA .{ }^{4} \mathrm{We}$ will use Knudsen diffusion as a hypothetical upper bound for the diffusion coefficient of gold assuming no potential energy gradients and assuming each gold cluster is an ideal gas particle. We do not claim that Knudsen diffusion mechanisms are at play in the micropores of TS-1, but merely provide this as a comparison. Additional discussion about using the Knudsen equation in MFItype zeolites is included in the Supporting Information. The Knudsen diffusion coefficient was calculated according to equation 5 , where $\mathrm{m}$ is the molar mass of gold, $\mathrm{d}$ is the pore diameter in TS-1 (here taken to be $5.5 \AA$ ), $\mathrm{R}$ is the ideal gas constant, and T is the temperature.

$$
D_{K}=\frac{d}{3} \sqrt{\frac{8 R T}{\pi m}}
$$

For the gold monomer, the Knudsen diffusion coefficient is $4.1 \times 10^{-8} \mathrm{~m}^{2} \mathrm{~s}^{-1}$, more than an order of magnitude higher than calculated using the NEB method. This would indicate the mass of gold is not so large that diffusion is implausible, at least for the monomer. The Knudsen diffusion equation does not include changes in van der Waals interactions due to intersections and channels which introduce enthalpic barriers. The Knudsen diffusivity of dimers is $2.92 \times 10^{-8} \mathrm{~m}^{2} \mathrm{~s}^{-1}$, further showing that it is the free energy barrier and not gold mass that have the dominant contribution to diffusivity. Trimer Knudsen diffusion coefficients are $2.4 \times 10^{-8} \mathrm{~m}^{2} \mathrm{~s}^{-1}$. 
While this analysis is simplistic and not relevant for TS-1, it does provide evidence that the gold diffusivity in a mesoporous material would not be negligible, and that gold diffusion can occur on rather fast timescales.

\section{S3 Additional References}

(1) F. Abouelnasr, M. K.; Smit, B. Diffusion in Confinement: Kinetic Simulations of Self- and Collective Diffusion Behavior of Adsorbed Gases. Physical Chemistry Chemical Physics 2012, 14 (33), 11600-11609. https://doi.org/10.1039/C2CP41147D.

(2) Voter, A. F.; Doll, J. D. Dynamical Corrections to Transition State Theory for Multistate Systems: Surface Self-diffusion in the Rare-event Regime. J. Chem. Phys. 1985, 82 (1), 8092. https://doi.org/10.1063/1.448739.

(3) Dubbeldam, D.; Beerdsen, E.; Vlugt, T. J. H.; Smit, B. Molecular Simulation of LoadingDependent Diffusion in Nanoporous Materials Using Extended Dynamically Corrected Transition State Theory. J. Chem. Phys. 2005, 122 (22), 224712. https://doi.org/10.1063/1.1924548.

(4) Kärger, J.; Ruthven, D. M. Diffusion in Zeolites and Other Microporous Solids; Wiley: New York, 1992. 